\title{
Immune complex-mediated antigen presentation induces tumor immunity
}

\author{
Khadija Rafiq, Amy Bergtold, and Raphael Clynes \\ Department of Medicine and Microbiology, Columbia University College of Physicians and Surgeons, New York, New York, USA
}

\begin{abstract}
Antigen uptake receptors on dendritic cells (DCs) provide efficient entry for the initiation of antigen-specific adaptive immunity. Here we show that targeting of antigen to Fc receptors on DCs accomplishes combined activation of Th1 CD4 and CD8 effector responses in vivo, namely delayedtype hypersensitivity and tumor immunity. Tumor immunity specific for ovalbumin-expressing tumors was provided by immunization with wild-type but not $F c \gamma R \gamma^{/-}$DCs loaded with ovalbumincontaining immune complexes. Tumor protection was eliminated when immune complex-loaded DCs lacked $\beta_{2}$ microglobulin, TAP, or MHC class II, demonstrating that Fc receptor-targeted antigenic uptake led to both MHC class I- and class II-restricted responses, which together are required for effector tumor immunity. Thus the cross-presentation pathway accessed by antigens acquired endocytically through $\mathrm{Fc}$ receptors links humoral and cellular immunity. These data suggest that administration of antitumor antibodies may enhance tumor-specific $T$ cell responses in vivo and provide the rationale for $\mathrm{Fc}$ receptor targeting in vaccine development.
\end{abstract}

This article was published online in advance of the print edition. The date of publication is available from the JCI website, http://www.jci.org. J. Clin. Invest. 110:71-79 (2002). doi:10.1172/JCI200215640.

\section{Introduction}

Adaptive tumor immunity is provided by collaborative MHC class II-restricted CD4 helper and MHC class I-restricted CD8 cytolytic effector responses. Thus, ideally, antigenic processing of tumor antigenic targets by antigen-presenting cells (APCs) should access both the MHC class I and MHC class II pathways. Yet, endocytosis or phagocytosis of extracellular antigens by antigen uptake receptors is processed primarily by APCs via the exogenous endosomal/lysosomal pathway, which ultimately delivers peptide onto surface MHC class II but not MHC class I molecules. However, CD8 ${ }^{+} \mathrm{MHC}$ class I responses can be initiated through the "crosspresentation pathway" $(1,2)$ via dendritic cells' (DCs') uptake of exogenous material, including transplanted (3), tumor (4-8), or virally infected cells (9). Previous efforts to exploit this cross-presentation pathway enabling access of extracellular antigens to the endogenous pathway have focused on DC phagocytosis of dying cells. Recent observations with in vitro systems $(10-12)$ suggest that uptake of antigen through Fc

Received for publication April 9, 2002, and accepted in revised form May 29, 2002

Address correspondence to: Raphael Clynes, Department of Medicine and Microbiology, Columbia University College of Physicians and Surgeons, P\&S 8-510, 630 West 168th Street, New York, New York 10032, USA. Phone: (212) 305-5289; Fax: (212) 305-1392; E-mail: rc645@columbia.edu.

Conflict of interest: No conflict of interest has been declared. Nonstandard abbreviations used: dendritic cell (DC); antigenpresenting cell (APC); immune complex (IC); antibody-dependent cell-mediated cytotoxicity (ADCC); ovalbumin (OVA); wild-type (WT); horseradish peroxidase (HRP); delayed-type hypersensitivity (DTH); bone marrow-derived dendritic cell (BMDC); sheep red blood cell (SRBC); Fc receptor (Fc $\gamma \mathrm{R})$. receptors (Fc $\gamma \mathrm{Rs}$ ) may represent an alternative method for cross-presentation. Schuurhuis (13) has shown that this pathway can induce antigen-specific CD8 responses in vivo, but the physiological relevance and potency of this pathway in effector immunity, including tumor immunity, have not yet been demonstrated.

The physiological consequences of cellular bound $\operatorname{IgG}$ and immune complexes (ICs) are mediated by FcyRs and include immunoregulatory and inflammatory responses. In particular, Fc $\gamma$ Rs on effector cells, including monocytes, neutrophils, mast cells, and NK cells, mediate phagocytosis, antibody-dependent cellmediated cytotoxicity (ADCC), and release of cytokines, chemokines, and other inflammatory mediators central to the protective and injurious properties of antibodies (14). Genetic evidence in humans $(15)$ and mice $(16,17)$ supports a general requirement for $\mathrm{Fc} \gamma \mathrm{R}$ engagement for the efficacy of antitumor antibodies in vivo, implicating Fc-dependent effector cell ADCC as a common mechanism underlying tumor-specific humoral immunity. However, a requirement for CD8 cellular cytotoxicity for the efficacy of an antitumor $\mathrm{mAb}$ (18) suggests that in addition to mediating ADCC, Fc $\gamma \mathrm{R}$-mediated enhancement of antigen presentation may also contribute to tumor immunity.

Capture of ICs by APCs likely contributes to the characteristic rapid appearance of high-titered antigen-specific IgGs during secondary exposures to antigen (19-21). This enhancement is due to the trapping, retention, and facilitated uptake of exogenous antigen by cellular complement and FcyRs on APCs and the subsequent intracellular targeting to the endosomal processing pathway for presentation onto membraneexpressed MHC class II molecules (22). Studies with in 
vitro systems have shown that $\mathrm{F} c \gamma \mathrm{R}$ uptake through the endosomal system, in addition to enhancing MHC class II antigenic processing and presentation, provides access, through undetermined mechanisms, to the cytosolic or endogenous pathway (10-12). Furthermore, in some systems, engagement of activating Fc $\gamma$ Rs induces DC activation and maturation (23). Thus, in vitro observations have suggested that antigenic targeting to FcyRs on DCs accomplishes several important aspects of $\mathrm{T}$ cell priming in cancer: facilitated uptake of antigen, class I and II presentation of antigen, and induction of DC activation and maturation. Our studies demonstrate, for the first time to our knowledge, that antigens acquired through endocytosis by DCs, in this case IC uptake, can facilitate tumor immunity in vivo, a situation likely reflective of the combined induction of $\mathrm{T}$ cell effector immunity resulting from Th1- and class I-restricted CD8 cell priming.

\section{Methods}

Mice. C57BL/6 mice and $\beta_{2} \mathrm{M}^{-/-}, \mathrm{TAP} 1^{-/-}$, and MHC class $I^{-/-}$C57BL $/ 6$ congenic mice were purchased from The Jackson Laboratory (Bar Harbor, Maine, USA). The $F c \gamma R \gamma^{-1}$ knockout mice were obtained from Taconic Farms (Germantown, New York, USA). The C57Bl.6 congenic OT-I and OT-II mice, carrying T cell receptor transgenes recognizing ovalbumin (OVA) peptides restricted to $\mathrm{H}-2^{\mathrm{b}} \mathrm{MHC}$ class I and class II, respectively, were provided by Janko Nikolic-Zugic (Oregon Health Sciences University, Portland, Oregon, USA) and Alan Frey (New York University School of Medicine, New York, New York, USA). All mice entered the study at 6 to 8 weeks old.

Cell lines. The MO- 4 cell lines are stable OVA cDNA transfectants of B16F10 (provided by Alan Houghton, Memorial Sloan-Kettering Cancer Center, New York, New York, USA), and B16 cell lines (a C57BL/6 melanoma cell line) were obtained from the American Type Culture Collection (Manassas, Virginia, USA). Cell culture media consisted of RPMI-1640 (Cellgro, Herndon, Virginia, USA) supplemented with $2 \mathrm{mM}$ L-glutamine, $100 \mathrm{U} / \mathrm{ml}$ penicillin, $100 \mu \mathrm{g} / \mathrm{ml}$ streptomycin, and $10 \%$ heat-inactivated fetal calf serum (FCS). Geneticin $(1 \mathrm{mg} / \mathrm{ml})$ was added every week for maintenance of selective pressure on stable transformants.

Antigens and other reagents. The $\mathrm{H}-2 \mathrm{~K}^{\mathrm{b}}$-restricted peptide SIINFEKL, corresponding to amino acid residues 257-264, and the $\mathrm{H}-2 \mathrm{D}^{\mathrm{b}}$-restricted 323-339 peptide (ISQAVHAAHAEINEAGR) of chicken OVA were synthesized by American Peptide Co. (Sunnyvale, California, USA) and by New England Peptide (Fitchburg, Massachusetts, USA) and highly purified (>95\%) as assessed by HPLC. Crystallized and lyophilized OVA was obtained from Worthington Biochemical Corp. (Lakewood, New Jersey, USA). The lyophilized rabbit IgG fraction to chicken albumin (anti-OVA) was purchased from Cappel ICN (Aurora, Ohio, USA). Peroxidase/antiperoxidase ICs were obtained from Sigma-Aldrich (St. Louis, Missouri, USA). All reagents were tested for measurable endotoxin activity with the Limulus Amebocyte Lysate kit (Biowhittaker, Walkersville, Maryland, USA) and were assessed directly for DC maturation activity. No reagent induced any change in the immunophenotype of DCs following 48 hours of incubation.

Generation of bone marrow DCs. The procedure used for the generation of DCs was that described by Lutz et al. (24). After removing all muscle tissues from the femurs and tibiae, the bones were placed in a $100-\mathrm{mm}$ dish with RPMI-1640. Both ends of the bones were cut with scissors in the dish, and then the marrow was flushed out using $2 \mathrm{ml}$ of RPMI-1640 with a syringe and 25gauge needle. The cell suspension was passed through nylon mesh to remove small pieces of bone and debris, and red cells were lysed with ammonium chloride. After washing, $2 \times 10^{5}$ cells were placed in a non-tissue-culture-coated Petri dish per ml of medium (RPMI, $10 \%$ FCS, penicillin, streptomycin, and L-glutamine) supplemented with either $20 \mathrm{ng} / \mathrm{ml} \mathrm{rGM-CSF}$ (PeproTech Inc., Rocky Hill, New Jersey, USA) or 10\% GM-CSF containing supernatant obtained from the GM-CSF transfectant J558L B cell hybridoma cell line (provided by Alan Houghton). At day 3 , equal volume of fresh medium and GM-CSF was added to the plate. At day 6, 50\% of the medium was aspirated and replaced with equal volume of fresh medium containing GM-CSF. DCs were harvested between days 7 and 10 of culture based on the morphological accumulation of medium-sized 10- to 50-cell DC aggregates, which appear at this time loosely attached to adherent cells. Immaturity of cells was confirmed by flow cytometric determination of low-level expression of both MHC class II and CD86. DCs were collected from plates by gentle aspiration and collection of nonadherent cells, which uniformly bore the DC immunophenotypic signature $\mathrm{CD} 11 \mathrm{c}^{+} \mathrm{CD} 14^{-}$.

\section{Endocytosis and phagocytosis assays}

Cellular immunofluorescence assessment of OVA uptake. Immature wild-type (WT) or $F c \gamma R \gamma^{/-}$bone marrowderived dendritic cells (BMDCs) were plated on lysinecoated coverslips and incubated with $10 \mu \mathrm{g} / \mathrm{ml}$ OVA alone, $50 \mu \mathrm{g} / \mathrm{ml}$ anti-OVA, or OVA-ICs $(50 \mu \mathrm{g} / \mathrm{ml}$ antiOVA and $10 \mu \mathrm{g} / \mathrm{ml}$ OVA) for 30 minutes. Cells were fixed in 4\% paraformaldehyde and permeabilized with $1 \%$ Triton X-100. Cells were blocked in PBS/1\% BSA, then stained with rabbit anti-OVA IgG (1:100 in PBS/1\% BSA), washed, and visualized with FITC-anti-rabbit IgG (1:100 in PBS/1\% BSA) using an E500 fluorescent microscope (Nikon Inc., Melville, New York, USA) and the RT SPOT digital camera/software package (Diagnostic Instruments, Sterling Heights, Michigan, USA).

Phagocytosis assays. $1 \%$ sheep red blood cells (SRBCs) were incubated with 1:500 dilutions of an IgG fraction of rabbit anti-SRBC (Cappel ICN) for 30 minutes at room temperature. Washed opsonized SRBCs were added to adherent BMDCs plated on tissue culture-coated glass slides (Becton Dickinson), Franklin Lakes, New Jersey, USA) 30 minutes earlier and incubated at $37^{\circ} \mathrm{C}$ for another 30 minutes. Unincorporated 
SRBCs were removed by rapid hypotonic lysis in water and fixed in $0.025 \%$ glutaraldehyde/PBS.

Adoptive transfer with antigen-pulsed DCs and tumor challenge. Donors BMDCs were derived from either syngeneic WT C57BL/ 6 mice or congenic C57Bl. $6 \beta_{2} \mathrm{M}^{-/-}$, TAP1 $1^{-/-}$, class $\mathrm{II}^{-/-}$, and $\mathrm{Fc} \gamma \mathrm{R} \gamma^{--}$mice. Immature DCs were cultured with OVA and anti-OVA (10 and 50 $\mu \mathrm{g} / \mathrm{ml}$, respectively) or OVA alone $(10 \mu \mathrm{g} / \mathrm{ml})$. The antigen-pulsed DCs were washed three times in PBS and administered to naive WT mice at a dose of $1 \times 10^{6}$ cells per mouse in $200 \mu \mathrm{l}$ PBS intravenously through the tail vein. One week later, $2 \times 10^{5}$ tumor cells (B16 or MO-4) were injected subcutaneously. Mice were monitored every 3 days for palpable tumors.

Cell proliferation assay. For restimulation of lymphocytes obtained after in vivo immunization, BMDC standard protocols were performed with a minor modification to reduce xenoprotein immunoreactivity upon restimulation with FCS in vitro. On day 6 of culture, bone marrow-derived DCs were removed from serum-containing media and cultured with fresh AIM V medium (GIBCO BRL; Life Technologies Inc., Grand Island, New York, USA) supplemented with $20 \mathrm{ng} / \mathrm{ml}$ GM-CSF for their final 6 days in culture prior to footpad immunization of recipients. Twelve days later, draining lymph node cells $\left(2 \times 10^{6}\right.$ per well $)$ were incubated in RPMI-1640 with 1\% FCS in 96-well flat-bottom microculture plates (Fisher Scientific Co., Pittsburgh, Pennsylvania, USA) in the presence of $10 \mu \mathrm{M}$ OVA-peptide I (amino acid residues 257-264) or 10 $\mu \mathrm{M}$ OVA-peptide II (amino acid residues 323-339). After 2 days, cells were pulsed with $1 \mu \mathrm{Ci}^{3} \mathrm{H}$-thymidine. Sixteen hours later, cells were harvested and thymidine incorporation was counted in a liquid scintillation counter. Proliferation of lymph node cells is expressed as the mean cpm of triplicate wells.

For in vitro stimulation of naive transgenic $\mathrm{T}$ cells, CD4 and CD8 T cells were positively selected with antiCD4 and anti-CD8 beads (Miltenyi Biotec Inc., Auburn, California, USA) CD4 and CD8 T cell splenocytes were cultured at a cell density of $2 \times 10^{6} / \mathrm{ml}$ with $1 \times 10^{5} / \mathrm{ml}$ pulsed DCs in 96-well flat-bottom microculture plates. At day 3 , cells were pulsed with $1 \mu \mathrm{Ci}{ }^{3} \mathrm{H}$-thymidine. Sixteen hours later, cells were harvested and thymidine incorporation was measured in a liquid scintillation counter. Proliferative responses are expressed as the mean cpm of triplicate wells.

For cytokine production, cells were plated at a cell density of $2 \times 10^{6} / \mathrm{ml}$ with $1 \times 10^{5} / \mathrm{ml}$ pulsed DCs in 24-well plates for 4 days prior to harvest of supernatants. For polyclonal activation, control $T$ cells were plated on anti-CD3-coated plates (Pharmingen, San Diego, California, USA) in the presence of $10 \mu \mathrm{g} / \mathrm{ml}$ anti-CD28 (Pharmingen).

Anti-OVA Ig ELISA. Blood was collected 15 days after primary immunization of mice with pulsed DCs. Sera were tested by ELISA for total IgG, IgG1, or IgG2a. Maxisorp plates (Fisher Scientific Co.) were coated overnight at $4^{\circ} \mathrm{C}$ with $10 \mu \mathrm{g} / \mathrm{ml} \mathrm{OVA}$ in coating buffer.
Fifty microliters of dilutions of both experimental samples and standard sera (obtained from mice immunized with OVA in CFA or Alum) were added to the plates and incubated overnight at $4^{\circ} \mathrm{C}$. Plates were then incubated for 2 hours at room temperature with $50 \mu \mathrm{l}$ of biotinylated anti-mouse IgG, IgG1, or IgG2a (diluted at 1:1,000) (Pharmingen), followed by incubation for 1 hour with $50 \mu \mathrm{l}$ avidin-horseradish peroxidase (avidin-HRP) (diluted at 1:10,000). Finally, $50 \mu \mathrm{l}$ 3,3',5,5'-tetramethylbenzidine dihydrochloride (SigmaAldrich) was added, and OD at $450 \mathrm{~nm}$ was measured 10-20 minutes later. ELISAs were standardized using sera obtained from mice immunized with OVA in either CFA or Alum. OVA-specific IgE was not detectable in any BMDC-immunized mice.

Cytokine concentrations in culture supernatants were determined with a sandwich ELISA technique, using Cytoscreen IL- 4 and IFN- $\gamma$ ELISA kits to measure different epitopes of each cytokine (BioSource International Inc., Camarillo, California, USA).

Delayed-type hypersensitivity responses. Seven days after primary immunization, the mice were challenged with $30 \mu \mathrm{g}$ OVA in $30 \mu \mathrm{l}$ PBS injected into the right footpad. The left footpad was injected with $30 \mu \mathrm{l}$ PBS alone. The thickness of the foot was measured with a Pocket Thickness Gauge (Mitumoyo Corp., Aurora, Illinois, USA) 24 hours after the challenge. Foot swelling as a result of OVA-specific delayed-type hypersensitivity (DTH) is expressed as the mean difference in swelling $(\mathrm{mm})$ between the OVA- and PBS-challenged feet.

\section{Results}

Immunization with IC-loaded DCs provides tumor immunity. To demonstrate the physiological significance of ICaugmented $\mathrm{T}$ cell priming to the initiation of a relevant immune response in vivo, the ability of IC-loaded DCs to induce tumor immunity was tested in prevention, treatment, and recall responses (Figure 1). C57BL/6 mice were injected intravenously with BMDCs alone, OVA-pulsed BMDCs, or OVA/IC-pulsed BMDCs. In tumor prevention studies, 7 days after DC immunization, MO- 4 cells (a syngeneic melanoma cell line transfected with the cDNA encoding OVA [ref. 25]) were injected subcutaneously into these mice (Figure 1a and Table 1) and mice were monitored for the development of palpable tumors. All mice developed large tumors when they were immunized with either BMDCs alone (9 of 10 tumor takes) or OVA-pulsed BMDCs (24 of 25 tumor takes). In contrast, only one tumor was palpable at 4 weeks in 25 mice immunized with OVA/IC-pulsed BMDCs. The OVA specificity of the antitumor immune response was confirmed since OVA/IC-pulsed BMDCs failed to offer any protection in mice injected with B16 melanoma cells (10 of 10 tumor takes) that did not express OVA. In the treatment studies, DC immunization was delayed until 7 days following injection with MO-4 cells. All mice receiving either BMDCs alone or BMDCs loaded with OVA developed tumors (10 of 10 

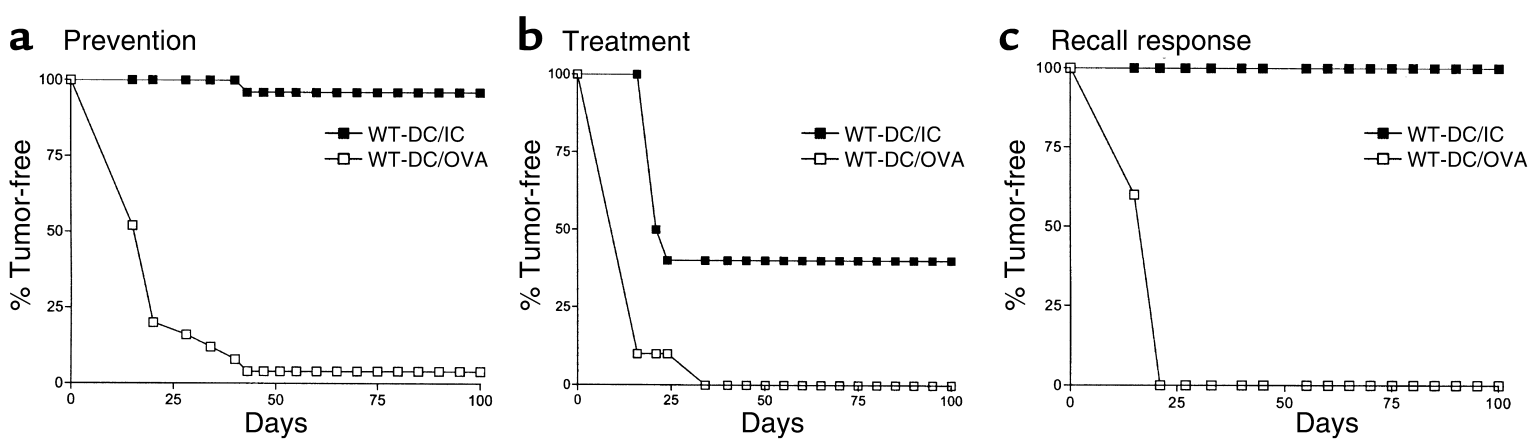

Figure 1

Immunization with BMDCs loaded with tumor antigen containing-ICs provides long-lasting and potent tumor immunity. (a) Prevention. Antigen-pulsed DCs $\left(1 \times 10^{6}\right.$ cells per mouse $)$ were administered intravenously to naive WT mice $(n=25$ per group $)$. One week later, $2 \times 10^{5}$ MO-4 cells were injected subcutaneously and mice were monitored for the development of palpable tumor. No protection was provided by immunization with OVA-loaded BMDCs; 24 of 25 mice developed tumors. Near-complete protection was seen after immunization with OVA/IC-loaded BMDCs (only 1 of 25 mice developed tumors). (b) Treatment. $2 \times 10^{5} \mathrm{MO}-4$ cells were injected subcutaneously; 1 week later, antigen-pulsed DCs $\left(1 \times 10^{6}\right.$ cells per mouse) were administered intravenously to naive WT mice $(n=10$ per group). (c) Recall response. Six months after intravenous immunization with antigen-pulsed BMDCs, WT mice $(n=5)$ were challenged subcutaneously with $2 \times 10^{5}$ MO-4 cells. No protection was provided by immunization with OVA-loaded BMDCs, but mice were fully protected 6 months after immunization with OVA/IC-loaded BMDCs. No significant differences in tumor protection were found between mice immunized with BMDCs alone and with BMDCs loaded with soluble OVA; for simplicity, the BMDC-alone group is not presented.

tumor takes in each group; Figure $1 \mathrm{~b}$ and data not shown). In contrast, treatment with BMDCs loaded with ICs cured $40 \%$ of mice bearing early-established tumors. Protection mediated by BMDC/IC immunization was long-standing and induced memory responses, as MO-4 tumor takes were prevented in all mice challenged with tumor 6 months after initial immunization with IC-loaded BMDCs (Figure 1c).

IC-mediated tumor immunity results from $F c R$ uptake of ICs and MHC class I and class II antigen presentation. To determine the mechanism of protection mediated by IC-loaded DCs, syngeneic DCs obtained from $F c \gamma R \gamma^{\prime-}$, $M H C$ class $\mathrm{II}^{-/-}, \mathrm{TAP1}^{-/-}$, and $\beta_{2} \mathrm{M}^{-/-}$mice were used for immunization (Table 1). Uptake through activation Fc $\gamma$ Rs was required for induction of tumor immunity, as use of $F c \gamma R \gamma^{\prime-}$ BMDCs loaded with ICs failed to induce tumor immunity. Thus, uptake of OVA-containing ICs through the remaining Fc $\gamma R s$, Fc $\gamma$ RII, does not result in $\mathrm{T}$ cell responses capable of rejecting tumors. We evaluated the contribution of both CD4 and CD8 responses to antitumor immunity by assessing recipients of IC-loaded class $\mathrm{II}^{-/-}, \beta_{2} \mathrm{M}^{-/-}$, and TAP1 ${ }^{-/-}$DCs for tumor protection. Tumor immunity induced by IC/BMDC vaccination required that DCs contain both an intact MHC class I and MHC class II antigen presentation pathway, demonstrating that full protection requires combined CD4 and CD8 cellular responses. The impaired ability of TAP1-/- or $\beta_{2} \mathrm{M}^{-/-}$ BMDCs to induce tumor immunity eliminates the possibility that recipient APCs were responsible for crosspresentation of transferred cells or material in vivo and instead confirms that transferred BMDCs were responsible for cross-priming MHC class I-restricted recipient $\mathrm{T}$ cells. Anti-OVA humoral responses were induced in recipients of IC-loaded TAP1 $1^{-/-}$and $\beta_{2} \mathrm{M}^{-/-}$BMDCs (data not shown), indicating that anti-OVA IgG alone was insufficient for protection. The impaired ability of MHC class $\mathrm{II}^{-/-}$BMDCs to provide tumor immunity implies an additional requirement for $\mathrm{CD} 4$ responses, although it does not distinguish between CD4-mediated contributions to either priming of OVA-specific CD8 cells (sensitization phase) or enhancement of the CD8 cytotoxic effector function (effector phase). Thus tumor immunity was significantly impaired in recipients of IC-loaded BMDCs harboring disrupted $M H C$ class II, $\beta_{2} M$, or TAP genes, consistent with a requirement for Fc $\gamma \mathrm{R}$-mediated access to both MHC class I and class II antigenic presentation pathways and for combined CD4 and CD8 T cell responses for antitumor effector immunity.

IC-loaded BMDCs induce antigen-specific humoral and CD4 and $C D 8$ cellular responses in vivo. To investigate the mechanism of protection further, $\mathrm{T}$ cell responses and antibody responses were directly assessed in recipient mice of BMDCs alone, OVA-pulsed BMDCs, or OVA/IC-pulsed BMDCs (Figure 2). Total IgG was not affected by any immunization (data not shown), but specific anti-OVA IgG responses were detectable in BMDCs loaded with OVA, and these IgG responses were significantly enhanced after immunization with OVA/IC-loaded BMDCs (Figure 2b). Strong Th2 responses were not apparent with BMDC immunization, as IgE specific for OVA was not detectable in any treatment group. To directly assess $\mathrm{T}$ cell priming in vivo, C57BL/6 mice were injected intravenously with DCs loaded with OVA or OVA/ICs. Two weeks later, lymph node cells were obtained from these animals and restimulated in vitro with OVA MHC class I- or class II-restricted immunodominant peptides for measurement of OVA-specific CD8 and CD4 responses. In this assay, $\mathrm{T}$ cells from naive mice are not activated and proliferative responses upon in vitro restimulation are 
indicative of in vivo priming. Mice that received DCs loaded with OVA or anti-OVA alone did not generate OVA-specific $\mathrm{T}$ cell responses. In contrast, $\mathrm{T}$ cells from mice that had received IC-loaded DCs had strong CD4 and CD8 OVA-specific proliferative responses upon restimulation with class II- and class I-restricted OVA peptides, respectively (Figure 2a). Thus, antigens acquired through Fc $\gamma \mathrm{R}$-mediated endocytosis by DCs can prime both $\mathrm{CD} 4$ and $\mathrm{CD} 8$ responses in vivo.

ICs induce DC maturation and activation of naive CD8 cells and $C D 4$ cells in vitro. Without exogenous inflammatory cytokines, bone marrow-derived DCs cultured for 10 days with GM-CSF are uniformly phenotypically immature and are highly endocytic and phagocytic, including through Fc $\gamma$ Rs. Amigorena and colleagues (10) reported that addition of ICs to these immature BMDC cultures resulted in cellular maturation and activation as seen by upregulation of costimulatory molecules (CD86, CD80, and CD40) and MHC class II. We similarly observed that OVA-containing ICs induced immuophenotypic maturation of WT but not activating FcyR-deficient cells (data not shown). Specifically, flow cytometric analysis revealed that 24 hours of IC stimulation of BMDCs led to increased surface expression of CD40, CD54, CD86, CD80, ICAM-1, and MHC class II. Neither OVA nor anti-OVA IgG alone enhanced expression of these immunophenotypic markers of maturation (data not shown) by either WT or $F c \gamma R \gamma^{\prime-}$ BMDCs. Interestingly, the upregulation of the adhesion molecule ICAM-1 (CD54) has been noted to be a Th1-driving influence $(26,27)$.

As seen in Figure 3, WT BMDCs exhibited efficient phagocytosis of antibody-opsonized SRBCs that was abrogated in $F c \gamma R \gamma^{\prime-}$ BMDCs, demonstrating a requirement for $\gamma$ chain signaling for IgG-mediated phagocytosis, as previously reported for macrophages (28). In contrast, endocytosis of OVA/ICs was comparable by both WT and $F c \gamma R \gamma^{\prime-}$ BMDCs. Thus, although endocytosis of ICs through FcyRII is efficient, it is not accompanied by the induction of maturation and does not lead to effector tumor immunity.

To evaluate the contribution of DC maturation to the enhanced antigen presentation mediated by ICs, OVA-specific $T$ cell receptor transgenic OT-I and OT-II naive OVA-specific $\mathrm{CD} 8^{+}$or $\mathrm{CD} 4^{+} \mathrm{T}$ cells, respectively, were cultured in vitro with BMDCs alone or loaded with OVA or OVA-containing ICs. BMDCs loaded with OVA-containing ICs stimulated potent CD4 and CD8 proliferative responses, demonstrating the ability of IC-loaded antigen to prime both class I- and class II-restricted naive $T$ cells (Figure 4a). Interestingly, this enhancement of antigen presentation appears specific for the antigen contained within the IC. Thus, addition of "irrelevant" HRP/rabbit anti-HRP (PAP) containing ICs to soluble OVA succeeded in inducing DC maturation (data not shown) but failed to enhance "bystander" OVA-specific class I- or II-restricted antigen presentation (Figure 4a). Thus, IC-mediated maturation is not likely to prime $\mathrm{T}$ cell responses to other self and non-self antigens to which the DC is exposed at the time of IC stimulation. Further, these data imply that DC maturation induced by ICs is not sufficient to fully explain their enhancement of $\mathrm{T}$ cell priming capacities. Other IC-mediated pathways must also contribute, including their facilitated entry into the antigen processing/presentation MHC class I and II pathways.

IC-loaded BMDCs induce Th1 responses in vitro and sensitize for DTH response in vivo. DCs critically regulate the initial steps in the immune response by providing a number of signals including chemokines and cytokines that polarize the developing $T$ cell response into either a Th1 or a Th2 pathway (29). As the development of a cytolytic CD8 response would be enhanced by a simultaneous polarized Th1 CD4 helper response $(30,31)$, we sought to determine whether IC-loaded DCs were functionally polarized to drive Th1, rather than Th2, $\mathrm{CD} 4$ responses by analysis of the $\mathrm{T}$ cell production of Th1 and Th2 cytokines (Figure 4b). Under nonpolarizing conditions, OT-II transgenic $\mathrm{CD} 4^{+} \mathrm{T}$ cells produced considerable amounts of both IL-4 and IFN- $\gamma$ when stimulated by $\alpha \mathrm{CD} 3 / \alpha \mathrm{CD} 28$, consistent with activation of a heterogenous population of both Th1- and Th2-polarized T cells. Immature BMDCs loaded with $10 \mu \mathrm{M}$ class II-restricted OVA peptide 323-339 preferentially induced the production of Th2 cytokines by stimulated OT-II cells. In contrast, stimulation of naive CD4 ${ }^{+}$OT-II T cells by IC-loaded BMDCs induced T cell activation and production of three times more IFN- $\gamma$ than IL-4, consistent with a Th1 polarized response. IFN- $\gamma$ was also elaborated by OT-I CD8 ${ }^{+} \mathrm{T}$ cells upon

Table 1

Tumor takes in recipients of OVA/IC-loaded BMDCs: tumor immunity requires MHC class I and II antigen presentation and activating Fc $\gamma$ Rs

\begin{tabular}{lcccccc}
\hline & & \multicolumn{2}{c}{ DC Genotype } \\
Loaded antigen & WT DCs/MO-4 tumors & $F c \gamma R \gamma^{--}$ & MHC class II-/- & TAP1-/- & $\beta_{2} M^{-/-}$ & WT DCs/B16 tumors \\
- & $9 / 10$ & $5 / 5$ & $5 / 5$ & $5 / 5$ & $5 / 5$ & $10 / 10$ \\
OVA & $24 / 25$ & $5 / 5$ & $5 / 5$ & $5 / 5$ & $5 / 5$ & ND \\
OVA/ICs & $1 / 25$ & $4 / 5$ & $4 / 5$ & $4 / 5$ & $4 / 5$ & $10 / 10$ \\
\hline
\end{tabular}

Mice were intravenously immunized with either $10^{6} \mathrm{BMDCS}$ alone or $10^{6} \mathrm{BMDC}$ loaded with either OVA or OVA/ICs, and then subcutaneously challenged 7 days later with $2 \times 10^{5} \mathrm{MO}-4$ B16 OVA transfectant cells. Recipients of WT DCs were challenged with either MO-4 cells (far left) or nontransfected B16 parental cells (far right). Data are expressed as the number of mice containing palpable tumors after 6 weeks over the total number of mice injected with tumor cells. IC-loaded WT BMDCs offered significant protection only for MO-4 tumors, not for B16 parental tumors. IC-loaded BMDCs lacking either activating Fc $\gamma$ receptors or MHC class II, TAP, or $\beta_{2} \mathrm{M}$ genes provided no significant protection compared with WT DCs $(P=0.0008$, Fisher's exact test). ND, not determined. 


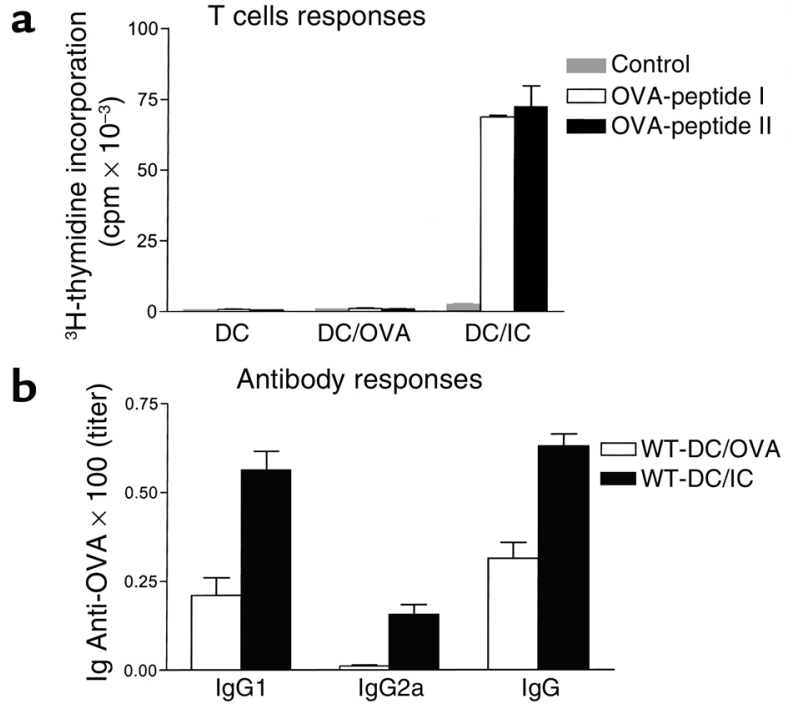

Figure 2

IC-loaded BMDCs induce OVA-specific cellular and humoral immunity in vivo. (a) OVA-peptide MHC class I- and II-restricted cellular immune responses result from OVA/IC-loaded BMDC immunization. Proliferative responses occur after addition of MHC class I- or class II-restricted peptides to lymph node cell populations from mice immunized with BMDCs loaded with OVA/ICs but not OVA alone. (b) Antibody responses are enhanced after immunization with OVA/IC-loaded BMDCs. OVA-specific ELISA reveals higher OVAspecific total $\lg G, \lg G 1$, and $\lg G 2 a$ titers in diluted sera from mice immunized 15 days earlier with IC-pulsed rather than OVA-pulsed BMDCs (ten mice per group)

activation during exposure to IC-loaded BMDCs, demonstrating that CD8 expansion and effector cell differentiation could be induced by IC-loaded BMDCs. In each case, intracellular staining confirmed that the cellular origin of IFN- $\gamma$ production were $\mathrm{CD}^{+}$or $\mathrm{CD} 8^{+}$ cells rather than cocultured CD $11 \mathrm{c}^{+}$DCs (data not shown). Notably, although minor proliferative responses were observed upon exposure of naive transgenic $T$ cells to BMDCs loaded with OVA alone, no detectable IFN- $\gamma$ production could be found in these cultures.

The effector phase of the DTH responses is the result of a Th1-driven inflammatory response (32) initiated upon a recall response to antigen. To address the relevance of the production of Th1 cytokines by OT-II cells in vitro to the situation in vivo, DTH responses were evaluated in normal mice sensitized with either BMDCs alone or BMDCs loaded with OVA or OVAcontaining ICs (Figure 5). Seven days after sensitization, recall responses were elicited by subcutaneous footpad challenge with soluble OVA. Inflammatory recall responses were not induced in recipients of BMDCs loaded with OVA alone. In contrast, all mice immunized with IC-loaded BMDCs developed footpad swelling, consistent with the induction of a Th1-polarized CD4 effector response in vivo. Thus, immunization with IC-loaded BMDCs induces both Th1 and CD8 effector immunity. Through engagement of Fc $\gamma$ Rs on DCs, antibody can induce CD4 and CD8 cellular effector immunity, and this suggests new strategies for the induction of tumor immunity.

\section{Discussion}

Passive immunization with antitumor antibodies represents a new class of therapeutics in the treatment of human malignancy. Mechanistically, efficacy of antitumor antibodies has been attributed to direct growthinhibitory effects of tumor cells, complement-mediated cytotoxicity, or ADCC resulting from Fc $\gamma$ R activation on myeloid and NK effector cells (33). Genetic evidence in humans $(15)$ and mice $(16,17)$ supports a general requirement for Fc $\gamma$ Rs engagement for the efficacy of antitumor antibodies in vivo, implicating Fc-dependent effector ADCC as a common mechanism underlying tumor-specific humoral immunity. However, a requirement for CD8 cellular cytotoxicity for the efficacy of an antitumor $\mathrm{mAb}(18,34)$ suggests that, in addition to mediation of ADCC, Fc $\gamma \mathrm{R}$-mediated enhancement of antigen presentation is another mechanism contributing to tumor immunity. The data presented here directly demonstrate that Fc $\gamma \mathrm{R}$ targeting of DCs results in tumor immunity through the activation of antigen-specific naive CD4 and CD8 T cells. Thus, an additional component of antitumor antibody efficacy, not previously appreciated, may be the induction of tumor antigen-specific $\mathrm{T}$ cell responses.

The priming of naive $T$ cells in vivo by extracellular antigen is orchestrated by DCs, which have multiple pathways for antigen uptake, including macropinocytosis, endocytosis, and phagocytosis (29). Processing of extracellular antigen via the exogenous pathway cul-

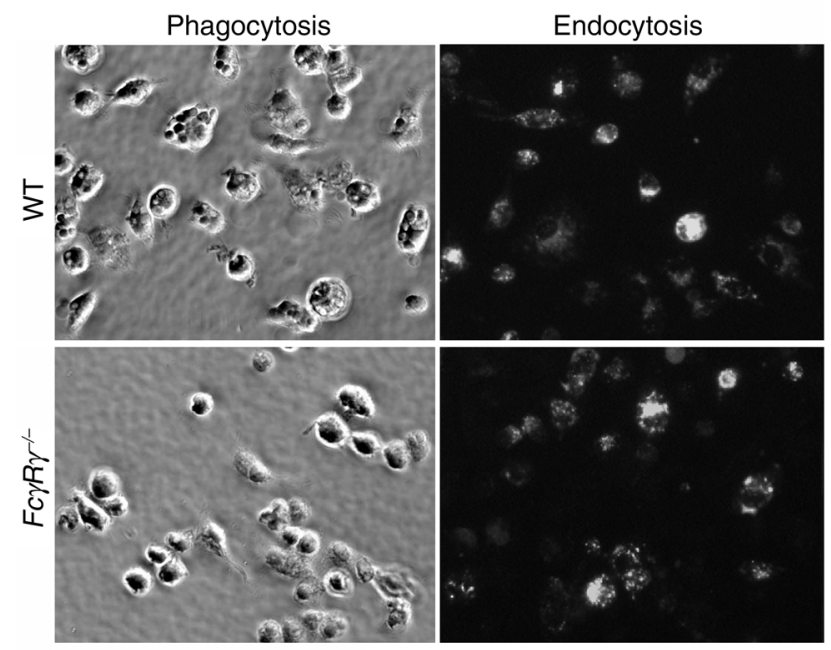

Figure 3

Phagocytic but not endocytic uptake of antibody-complexed antigen requires activating Fc $\gamma R$ s on DCs. Immature BMDCs were incubated for 30 minutes with either OVA-containing ICs for endocytosis or antibody-opsonized SRBCs for phagocytosis. Uptake of soluble OVA or anti-OVA alone was negligible (data not shown), while uptake of OVA/ICs was dramatically enhanced for both WT and FcYR $\gamma^{-1}$ BMDCs, indicating that inhibitory FcRs are capable of efficiently endocytosing OVA/ICs. In contrast, phagocytic uptake of antibodyopsonized SRBCs was seen in WT but not in FcYR $\gamma^{-/}$BMDCs. 
minates in the presentation of antigenic peptides on MHC class II for the activation of CD4 helper T cell responses and humoral responses in vivo (35-37). Indeed, our data are consistent with other studies (19, $38-40)$ in demonstrating that DC uptake of immunecomplexed antigen through activating Fc $\gamma \mathrm{R}$-mediated endocytosis results in augmented CD4 priming in vitro and in vivo. Furthermore, IC-loaded BMDCs preferentially polarized Th1 effector cell differentiation of naive OT-II transgenic CD4 cells. The quality of the CD4 effector Th1-polarized response in vivo is demonstrated by the DTH responses seen in OVAchallenged BMDC OVA/IC-sensitized mice. The DTH response, dependent on CD4 cell elaboration of Th1 cytokines, was abrogated in $F c \gamma R \gamma^{--}$mice (38), but this previous study could not distinguish between require-

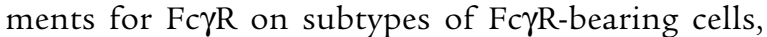
including APCs and cellular effectors during sensitization and/or challenge phases. Our data suggest that APC expression of activating Fc $\gamma$ Rs contributes to the induction of antibody-enhanced Th1 responses.

In addition to enhancing CD4 responses, $\mathrm{Fc} \gamma \mathrm{R}$ targeting accomplishes a goal of effective tumor immunization, namely the induction of potent CD8 effector immunity specific for tumor-specific antigens. Recent in vitro studies have shown that DC Fc $\gamma \mathrm{R}$ targeting of soluble ICs (10) and opsonized human tumor cells (11) can facilitate class I-restricted antigen presentation to antigen-experienced CD8 T cells. In our studies we show that DC uptake of ICs can directly induce the proliferation and activation of effector cell differ- entiation of MHC class I-restricted naive transgenic OT-I CD8 cells. Tumor protection studies showed that protective immunity required DCs to harbor intact MHC class I and II antigen presentation pathways. This eliminates the possibility that transferred DCs were cross-presented by host DCs and demonstrates that the Fc $\gamma \mathrm{R}$-mediated cross-presentation pathway is required for $\mathrm{CD} 8$ responses in vivo. Further, a requirement for DC MHC class II expression suggests that both CD4 and CD8 responses collaborate in vivo for full tumor protection. Our data cannot distinguish whether IC-induced Th1 CD4 responses collaborate with CD8 cellular cytotoxicity by providing IFN- $\gamma$ to augment CD8 responses during sensitization (41) and/or at the effector level $(30,31)$. Thus, Fc $\gamma R$ targeting has the potential to accomplish several important features for vaccine design; efficient antigen uptake, and processing of antigen for both MHC class I and class II antigenic presentation for priming of both CD4 and CD8 cells.

IC-loaded BMDCs induced potent and long-lasting effector tumor immunity in vivo, curing $40 \%$ of earlyestablished MO-4 tumors and providing effector memory responses for at least 6 months. Tumor immunity was antigen-specific, as no protection was seen when using the parental cell line B16F10, which lacks OVA expression. This dismisses the possibility that presentation of FCS-derived xenogenic antigens by cultured DCs is solely responsible for tumor immunity; however, xenogenic responses may still contribute to rejection of OVA-expressing tumors (42).

\section{Figure 4}

OVA/IC-mediated antigen presentation in vitro induces Th1 CD4 and CD8 cellular proliferation and effector differentiation of naive transgenic T cells. (a) Anti-OVA $T$ cell responses. IC-mediated antigen presentation efficiently drives OT-I and OT-II proliferative responses in vitro. $2 \times 10^{5}$ CD4 OT-II and $4 \times 10^{5}$ CD8 OT-I cells were cultured with $10^{4}$ or $2 \times 10^{4}$ antigen-pulsed BMDCs in 96 -well culture plates. ${ }^{3} \mathrm{H}$-thymidine incorporation was measured between 36 and 48 hours later and expressed in cpm. OVA $(10 \mu \mathrm{g} / \mathrm{ml})$, OVA-containing $\mathrm{ICs}(10 \mu \mathrm{g} / \mathrm{ml}$ OVA and $50 \mu \mathrm{g} / \mathrm{ml}$ anti-OVA), or OVA $(10 \mu \mathrm{g} / \mathrm{ml})$ plus "irrelevant" peroxidase/antiperoxidase (PAP) complexes $(50 \mu \mathrm{g} / \mathrm{ml})$ were added to DCs for 48 hours prior to incubation with $T$ cells. The results are representative of five separate experiments. (b) Cytokine production. IC-loaded BMDCs induce IFN- $\gamma$ production by naive OT-I and OT-II transgenic cells. CD4 OT-II or CD8 OT-I transgenic T cells were cultured at $10^{6} / \mathrm{ml}$ with $5 \times 10^{4} / \mathrm{ml}$ pulsed DCs in 24-well plates. IL- 4 and IFN- $\gamma$ production was determined by ELISA after 3-5 days of culture. The results are from two independent experiments. The sensitivity of the ELISAs were $1 \mathrm{pg} / \mathrm{ml}$. Culture of OT-I or OT-II transgenic T cells with DCs alone (i.e., without OVA peptides or soluble OVA) did not elaborate detectable IFN- $\gamma$ or IL-4.

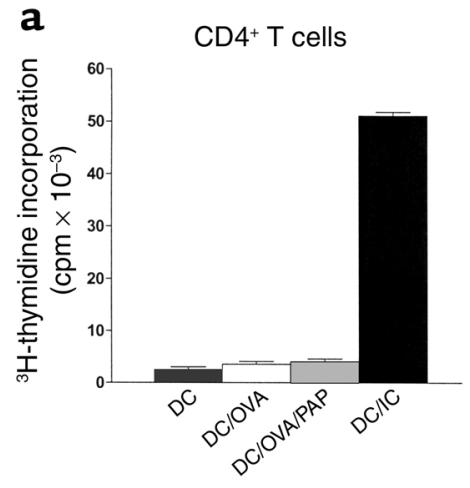

b

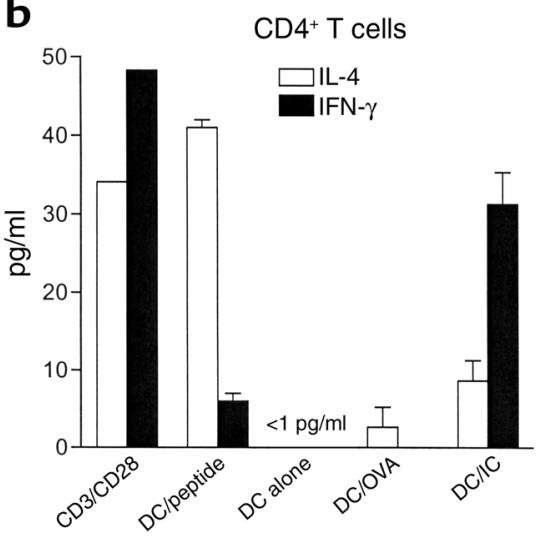

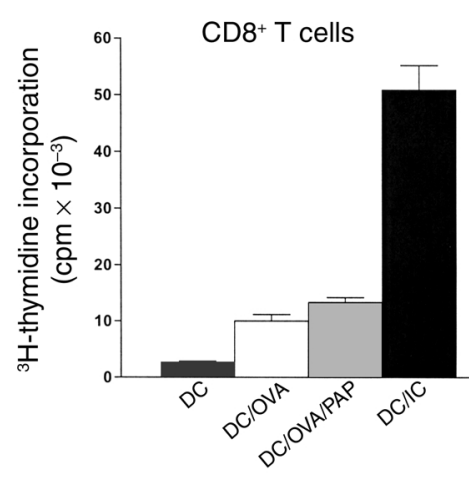

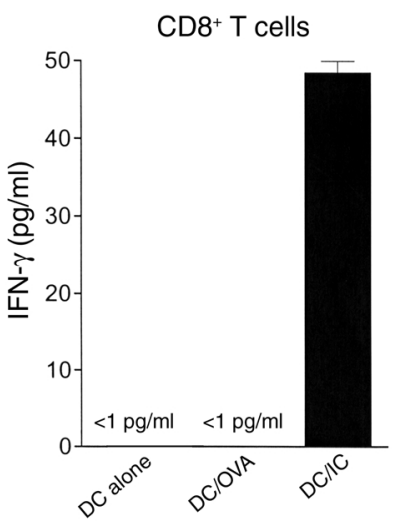




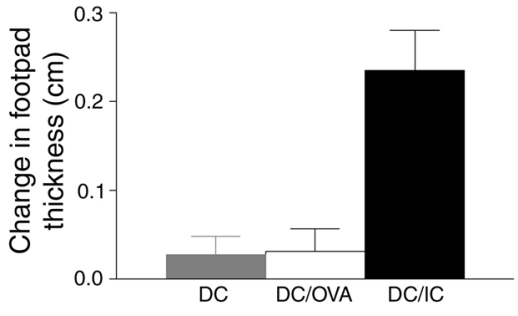

\section{Figure 5}

Sensitization with IC-loaded BMDCs induces DTH effector responses. Groups of eight to ten mice were immunized with $1 \times 10^{6}$ cells per mouse of OVA-pulsed DCs or IC-pulsed DCs in the front footpad. One week after primary immunization (sensitization), the mice were challenged with PBS in one footpad and OVA in the other footpad. Change in footpad thickness (OVA-injected footpad vs. PBS-injected control footpad) was measured 9 days after initial immunization. Error bars are the SEM of each group.

Interestingly, this enhancement of antigen presentation appears specific for the antigen contained within the IC. The addition of "irrelevant" HRP/anti-HRP containing ICs induced DC maturation but did not augment the presentation of soluble "bystander" OVA or immunodominant OVA-derived peptides (Figure 4 and data not shown). Thus, DC maturation induced by ICs is not sufficient to fully explain their enhancement of $\mathrm{T}$ cell priming capacities. Other IC-mediated pathways must also contribute, including their facilitated entry into the activating Fc $\gamma R$ antigen presentation pathways for MHC class I and class II. Others have noted that DC maturation results in an initial burst of antigen processing followed by a rapid shut-down of further processing. This results in a "snapshot" display of antigenic peptides synchronous with the initiation of the maturation stimulus (25). However, this display, even if temporally constrained, would likely result in the display and potential $\mathrm{T}$ cell priming of many stimulus-unrelated peptides, including those with selfspecificities, if the processing were not further restricted. In the case of ICs it appears that the enhancement of antigen processing is coupled to the antigen within the IC, thus limiting extraneous and perhaps injurious priming of other irrelevant self and non-self antigens. Thus, IC-loaded DCs induce both CD4 and CD8 priming in vitro, in a manner specific for the antigen contained within the IC.

While activating Fc $\gamma$ Rs are required for tumor immunity, the data presented here do not distinguish between potential roles for the two activating Fc $\gamma$ Rs, types I and III. Previous studies have demonstrated that targeting Fc $\gamma R$ I can result in enhanced humoral responses $(40,43$, 44), but class I-restricted responses were not evaluated. Recent in vitro studies with $F c \gamma R I^{-/-}$- and $F_{c} \gamma R I I I^{-/-}$-deficient BMDCs have suggested a role for both of these receptors in IC-mediated cross-presentation (45). Furthermore, while uptake by FcyRII alone was insufficient to induce tumor responses, a potential role for this inhibitory receptor (46) in modulating DC function remains to be determined. Fc $\gamma \mathrm{RII}$ is highly expressed on immature BMDCs and efficiently endocytoses ICs (Figure 3). Entry of ICs through Fc $\gamma$ RII may lead to an endosomal processing pathway distinct from that accessed by the $\gamma$-associated Fc $\gamma R$ I and Fc $\gamma R I I I$ receptors (47). Preliminary work in our laboratory suggests that IC targeting to lysosomes occurs rapidly after entry by Fc $\gamma \mathrm{RI}$ and III but not after entry by Fc $\gamma$ RII. Indeed, the comparable level of intracellular ICs seen in WT BMDCs (which express all three FcyRs) and $F c \gamma R \gamma^{\prime-}$ BMDCs (which express only FcyRII) is consistent with a delay in degradation and resultant accumulation of ICs in Fc $\gamma$ RII-bearing $F c \gamma R^{-/-}$BMDCs.

Thus, $\mathrm{Fc} \gamma \mathrm{R}$ targeting of antigen provides BMDCs the ability to prime both CD4 and CD8 responses in vivo. The ability of antigen-specific antibody to complex antigen and facilitate its uptake and retention by APCs is well described for the enhancement of MHC class II-restricted responses during secondary responses. The data presented here are consistent with the notion that antigen-containing ICs may also facilitate the activation of MHC class I-restricted responses by CD8 cells. Thus, antibody formed during a primary immune response would augment the potential for CD8-mediated cellular immunity upon secondary exposure to antigen, thus linking humoral and cellular immunity to tumor, auto-, allo-, or microbial antigens. Regarding tumor immunity, our data suggest that antitumor antibody-mediated efficacy may result from antibody-mediated enhancement of tumor antigen presentation to $T$ cells, suggesting alternative approaches to improving efficacy. Finally, activating Fc $\gamma$ R targeting may represent a novel approach for vaccine design for active immunization.

\section{Acknowledgments}

R. Clynes was funded in part by the National Cancer Institute, the Sidney Kimmel Foundation for Cancer Research, and by the Speaker's Fund for Biomedical Research: Toward the Science of Patient Care, awarded by the City of New York. We would like to thank Anamika Gavhane for technical assistance and Ralph Steinman for his encouragement and advice.

1. Heath, W.R., and Carbone, F.R. 2001. Cross-presentation, dendritic cells, tolerance and immunity. Annu. Rev. Immunol. 19:47-64.

2. Carbone, F.R., and Bevin, M.J. 1990. Class-I restricted processing and presentation of exogenous cell associated antigen in vivo. J. Exp. Med. 171:377-387.

3. Huang, A.Y., Bruce, A.T., Pardoll, D.M., and Levitsky, H.I. 1996. In vivo cross-priming of MHC class I-restricted antigens requires the TAP transporter. Immunity. 4:349-355.

4. Berard, F., et al. 2000. Cross-priming of naive CD8 T cells against melanoma antigens using dendritic cells loaded with killed allogeneic melanoma cells. J. Exp. Med. 192:1535-1544.

5. Jenne, L., Arrighi, J.F., Jonuleit, H., Saurat, J.H., and Hauser, C. 2000. Dendritic cells containing apoptotic melanoma cells prime human CD8+ T cells for efficient tumor cell lysis. Cancer Res. 60:4446-4452.

6. Hoffmann, T.K., Meidenbauer, N., Dworacki, G., Kanaya, H., and Whiteside, T.L. 2000. Generation of tumor-specific T-lymphocytes by crosspriming with human dendritic cells ingesting apoptotic tumor cells. Cancer Res. 60:3542-3549.

7. Hoffmann, T.K., Meidenbauer, N., Muller-Berghaus, J., Storkus, W.J., and Whiteside, T.L. 2001. Proinflammatory cytokines and CD40 ligand enhance cross-presentation and cross-priming capability of human dendritic cells internalizing apoptotic cancer cells. J. Immunother. 24:162-171. 
8. Nouri-Shirazi, M., et al. 2000. Dendritic cells capture killed tumor cells and present their antigens to elicit tumor-specific immune responses. J. Immunol. 165:3797-3803.

9. Albert, M.L., Sauter, B., and Bhardwaj, N. 1998. Dendritic cells acquire antigen from apoptotic cells and induce class I-restricted CTLs. Nature. 392:86-89.

10. Regnault, A., et al. 1999. Fcgamma receptor-mediated induction of dendritic cell maturation and major histocompatibility complex class Irestricted antigen presentation after immune complex internalization. J. Exp. Med. 189:371-380.

11. Dhodapkar, K.M., Krasovsky, J., Williamson, B., and Dhodapkar, M.V. 2002. Antitumor monoclonal antibodies enhance cross-presentation of cellular antigens and the generation of myeloma-specific killer $\mathrm{T}$ cells by dendritic cells. J. Exp. Med. 195:125-133.

12. Machy, P., Serre, K., and Leserman, L. 2000. Class I-restricted presentation of exogenous antigen acquired by Fcgamma receptor-mediated endocytosis is regulated in dendritic cells. Eur. J. Immunol. 30:848-857.

13. Schuurhuis, D.H., et al. 2002. Antigen-antibody immune complexes empower dendritic cells to efficiently prime specific CD8+ CTL responses in vivo. J. Immunol. 168:2240-2246.

14. Ravetch, J.V., and Clynes, R.A. 1998. Divergent roles for Fc receptors and complement in vivo. Annu. Rev. Immunol. 16:421-432.

15. Cartron, G., et al. 2002. Therapeutic activity of humanized anti-CD20 monoclonal antibody and polymorphism in IgG Fc receptor FcgammaRIIIa gene. Blood. 99:754-758.

16. Clynes, R., Takechi, Y., Moroi, Y., Houghton, A., and Ravetch, J.V. 1998. Fc receptors are required in passive and active immunity to melanoma. Proc. Natl. Acad. Sci. USA. 95:652-656.

17. Clynes, R.A., Towers, T.L., Presta, L.G., and Ravetch, J.V. 2000. Inhibitory Fc receptors modulate in vivo cytoxicity against tumor targets. Nat. Med. 6:443-446.

18. Vasovic, L.V., Dyall, R., Clynes, R.A., Ravetch, J.V., and Nikolic-Zugic, J. 1997. Synergy between an antibody and CD8+ cells in eliminating an established tumor. Eur. J. Immunol. 27:374-382.

19. Heyman, B. 2000. Regulation of antibody responses via antibodies, complement, and Fc receptors. Annu. Rev. Immunol. 18:709-737.

20. Cohen, B.E., Rosenthal, A.S., and Paul, W.E. 1973. Antigen-macrophage interaction. II. Relative roles of cytophilic antibody and other membrane sites. J. Immunol. 111:820-828.

21. Bachmann, M.F., Kundig, T.M., Hengartner, H., and Zinkernagel, R.M. 1994. Regulation of IgG antibody titers by the amount persisting of immune-complexed antigen. Eur. J. Immunol. 24:2567-2570.

22. Heyman, B. 1990. The immune complex: possible ways of regulating the antibody response. Immunol. Today. 11:310-313.

23. Regnault, A., et al. 1999. Fcgamma receptor-mediated induction of dendritic cell maturation and major histocompatibility complex class Irestricted antigen presentation after immune complex internalization. J. Exp. Med. 189:371-380.

24. Lutz, M.B., et al. 1999. An advanced culture method for generating large quantities of highly pure dendritic cells from mouse bone marrow. J. Immunol. Methods. 223:77-92.

25. Turley, S.J., et al. 2000. Transport of peptide-MHC class II complexes in developing dendritic cells. Science. 288:522-527.

26. Salomon, B., and Bluestone, J.A. 1998. LFA-1 interaction with ICAM-1 and ICAM-2 regulates Th2 cytokine production. J. Immunol. 161:5138-5142.
27. Bleijs, D.A., de Waal-Malefyt, R., Figdor, C.G., and van Kooyk, Y. 1999. Costimulation of T cells results in distinct IL-10 and TNF-alpha cytokine profiles dependent on binding to ICAM-1, ICAM-2 or ICAM-3. Eur. J. Immunol. 29:2248-2258.

28. Takai, T., Li, M., Sylvestre, D., Clynes, R., and Ravetch, J.V. 1994. FcR gamma chain deletion results in pleiotrophic effector cell defects. Cell. 76:519-529.

29. Banchereau, J., et al. 2000. Immunobiology of dendritic cells. Annu. Rev. Immunol. 18:767-811.

30. Pardoll, D.M., and Topalian, S.L. 1998. The role of CD4+ T cell responses in antitumor immunity. Curr. Opin. Immunol. 10:588-594.

31. Toes, R.E., Ossendorp, F., Offringa, R., and Melief, C.J. 1999. CD4 T cells and their role in antitumor immune responses. J. Exp. Med. 189:753-756.

32. Abbas, A.K., Murphy, K.M., and Sher, A. 1996. Functional diversity of helper T lymphocytes. Nature. 383:787-793.

33. Carter, P. 2001. Improving the efficacy of antibody-based cancer therapies. Nature Rev. Cancer. 1:118-127.

34. Honeychurch, J., et al. 2000. Therapeutic efficacy of FcgammaRI/CD64directed bispecific antibodies in B-cell lymphoma. Blood. 96:3544-3552.

35. Mellman, I., and Steinman, R.M. 2001. Dendritic cells: specialized and regulated antigen processing machines. Cell. 106:255-258.

36. Amigorena, S., and Bonnerot, C. 1999. Fc receptor signaling and trafficking: a connection for antigen processing. Immunol. Rev. 172:279-284.

37. Amigorena, S., and Bonnerot, C. 1999. Fc receptors for IgG and antigen presentation on MHC class I and class II molecules. Semin. Immunol. 11:385-390.

38. Hamano, Y., Arase, H., Saisho, H., and Saito, T. 2000. Immune complex and $\mathrm{Fc}$ receptor-mediated augmentation of antigen presentation for in vivo Th cell responses. J. Immunol. 164:6113-6119.

39. Heijnen, I.A., et al. 1996. Antigen targeting to myeloid-specific human Fc gamma RI/CD64 triggers enhanced antibody responses in transgenic mice. J. Clin. Invest. 97:331-338.

40. Keler, T., et al. 2000. Targeting weak antigens to CD64 elicits potent humoral responses in human CD64 transgenic mice. J. Immunol. 165:6738-6742.

41. Bennett, S.R., Carbone, F.R., Karamalis, F., Miller, J.F., and Heath, W.R. 1997. Induction of a CD8+ cytotoxic T lymphocyte response by crosspriming requires cognate CD4+ T cell help. J. Exp. Med. 186:65-70

42. Schnell, S., Young, J.W., Houghton, A.N., and Sadelain, M. 2000. Retrovirally transduced mouse dendritic cells require CD4+ T cell help to elicit antitumor immunity: implications for the clinical use of dendritic cells. J. Immunol. 164:1243-1250.

43. Gosselin, E.J., et al. 1992. Enhanced antigen presentation using human Fc gamma receptor (monocyte/macrophage)-specific immunogens. J. Immunol. 149:3477-3481.

44. Guyre, P.M., et al. 1997. Increased potency of Fc-receptor-targeted antigens. Cancer Immunol. Immunother. 45:146-148.

45. Ioan-Facsinay, A., et al. 2002. FcgammaRI (CD64) contributes substantially to severity of arthritis, hypersensitivity responses, and protection from bacterial infection. Immunity. 16:391-402.

46. Ravetch, J.V., and Lanier, L.L. 2000. Immune inhibitory receptors. Science. 290:84-89.

47. Amigorena, S., et al. 1998. Type II and III receptors for immunoglobulin $\mathrm{G}(\mathrm{IgG})$ control the presentation of different $\mathrm{T}$ cell epitopes from single IgG-complexed antigens. J. Exp. Med. 187:505-515. 